LETTER TO THE EDITOR

\title{
Automictic parthenogenesis and rate of transition to homozygosity
}

Heredity (2011) 107, 187-188; doi:10.1038/hdy.2010.172; published online 19 January 2011

The production of diploid daughters from unfertilized eggs can be achieved through various cytological mechanisms. Under apomictic parthenogenesis, meiosis is suppressed, and the absence of recombination or re-assortment of alleles leads to the production of a 'true clone' of the mother. By contrast, under automictic parthenogenesis, the first stages of meiosis are conserved and diploidy is restored by fusion of two nuclei originating from the same primary oocyte (terminal, central or random fusion) or by gamete duplication (Suomalainen et al., 1987). Automictic parthenogenesis increases homozygosity at each generation, but the rate of increase depends on the cytological mechanism restoring diploidy.

In a previous paper in Heredity, we determined the mode of thelytokous parthenogenesis in the ant Cataglyphis cursor, by comparing the observed generational increase in homozygosity at several informative loci with theoretical expectations (Table 1 in Pearcy et al., 2006). We argued that the expected rate of transition from heterozygosity to homozygosity at each generation was
0.33 (rather than 0.50) for loci with high recombination under terminal and central fusion. Here we clarify the reasoning behind the calculations.

During gametogenesis, the duplicated homologous chromosomes of the $4 n$ primary oocyte pair at the prophase of meiosis I and crossovers can occur between chromatids (Figure 1a). The probability of crossover in the region between the centromere and a given locus increases with the distance between the two. However, when multiple crossovers occur, the original location of the alleles can, by chance, be restored (locus 2 in Figure 1b). Also, if alleles are swapped for both chromatid pairs, the effects of the two recombination events cancel out each other (locus 2 in Figure 1c). In short, the probability that the alleles are effectively swapped between homologous chromosomes is not equal to the probability of observing at least one crossover.

In theory, when the distance between a given locus and the centromere is maximum (that is, infinite), crossovers are so frequent that one may consider that alleles segregate independently. In this case, each offspring of a $[a b]$ heterozygous mother producing $\left[a_{1} a_{2} b_{1} b_{2}\right]$ $4 n$ primary oocytes has a $1 / 3$ probability of being homozygous $\left[a_{1} a_{2}\right]$ or $\left[b_{1} b_{2}\right]$. For example, if the chroma-

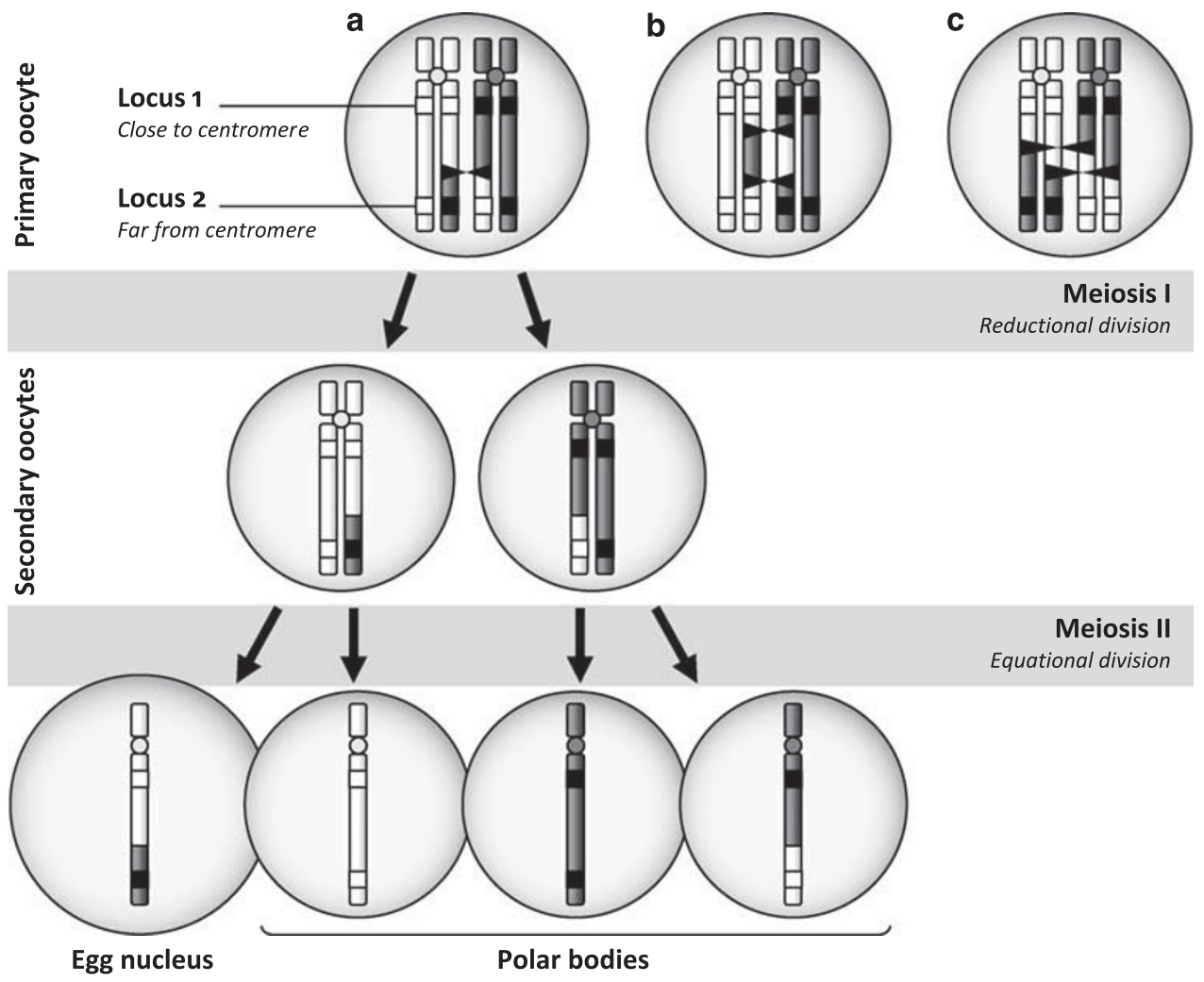

Figure 1 Effects of recombination during oogenesis. Chromosomal crossovers (black triangles) result in alleles being swapped between homologous chromatids (a), leading to alternate allele segregation in the egg nucleus and the polar bodies. However, multiple crossovers may, by chance, restore the original location of alleles (b) or neutralize each other (c). 
tid in the egg nucleus bears allele $a_{1}$, there is 1 chance out of 3 that fusion will occur with a polar body possessing the allele $a_{2}$ rather than $b_{1}$ or $b_{2}$. Of course, the same reasoning holds for the other alleles. Interestingly, this probability of transition to homozygosity is independent of the mode of fusion (terminal, central or random), as alleles segregate randomly from the primary oocyte to the polar bodies.

Thus, effective recombination probability for any given locus is lower than the rate of crossover and, in theory, should never reach 1 . Under automictic parthenogenesis with terminal, central or random fusion, extreme rate of crossover should result in alleles segregating independently. For these loci, the rate of transition to homozygosity is equal to the statistical probability of picking two identical items among two pairs without replacement, that is $1 / 3$.

\section{Conflict of interest}

The authors declare no conflict of interest.

\section{Acknowledgements}

This work was supported by grants from the Belgian Fond National de la Recherche Scientifique-FNRS (SA and $\mathrm{OJH}$ ) and the Fonds pour la Recherche dans l'Industrie et l'Agriculture-FRIA (MP).

M Pearcy, OJ Hardy and S Aron Evolutionary Biology and Ecology Unit, Université Libre de Bruxelles, Brussels, Belgium E-mail: mpearcy@ulb.ac.be

\section{References}

Pearcy M, Hardy OJ, Aron S (2006). Thelytokous parthenogenesis and its consequences on inbreeding in an ant. Heredity 96: 377-382.

Suomalainen E, Saura A, Lokki J (1987). Cytology and Evolution in Parthenogenesis. CRC Press Inc.: Boca Raton, FL. 